\title{
Test performance of optical coherence tomography angiography in detecting retinal diseases: a systematic review and meta-analysis
}

\author{
Livia Faes ${ }^{1,2}$ - Nicolas S. Bodmer ${ }^{3}$. Silvan Locher ${ }^{1} \cdot$ Pearse A. Keane $\mathbb{1}^{2,4} \cdot$ Konstantinos Balaskas $^{2}$. \\ Lucas M. Bachmann ${ }^{3} \cdot$ Reinier O. Schlingemann ${ }^{5} \cdot$ Martin K. Schmid $^{1}$
}

Received: 18 July 2018 / Revised: 22 December 2018 / Accepted: 7 February 2019 / Published online: 10 April 2019

(c) The Royal College of Ophthalmologists 2019

\begin{abstract}
Objective To investigate the diagnostic accuracy of optical coherence tomography angiography (OCTA) in detecting vascular characteristics of chorio-retinal disease.

Methods Evidence acquisition: We searched Web of Science, Scopus, and Medline by the citation of references and complemented these electronic searches by checking the list of references of included and review articles. Screening, selection, assessment, and extraction was performed in parallel by two authors.

Results Evidence synthesis: Systematic review and exploratory meta-analysis. The ten studies that contributed to the metaanalysis enrolled 440 eyes and allowed constructing ten two-by-two tables. The tables reported on detection of choroidal neovascularization (CNV) in eyes suffering from either age-related macular degeneration (4), central serous chorioretinopathy (2), myopia (2), foveomacular vitelliform dystrophy (1), or a mixed cohort suffering from multiple retinal diseases (1). Of the ten studies, six used a cohort and four a case-control design. We found a pooled sensitivity of 0.90 (95\% confidence intervals (CIs): $0.82-0.95$ ) and a pooled specificity of 0.97 (95\% CI: 0.89-0.99). Corresponding positive and negative likelihood ratios were 32.3 (95\% CI: 7.4-141.6) and 0.10 (95\% CI: 0.06-0.20), respectively. No pooling was possible for retinal vascular parameters of diabetic retinopathy, polypoidal choroidal vasculopathy, or detection of CNV activity.

Conclusions The results of highly biased and heterogeneous studies assessing the diagnostic performance of OCTA highlight the need for further analyses of methodologically sound and sufficiently sized clinical evaluations.
\end{abstract}

These authors contributed equally: Livia Faes, Nicolas S Bodmer

Supplementary information The online version of this article (https:// doi.org/10.1038/s41433-019-0421-3) contains supplementary material, which is available to authorized users.

Nicolas S. Bodmer

bodmer@medignition.ch

Eye Clinic, Cantonal Hospital of Lucerne, Lucerne, Switzerland

2 Medical Retina Department, Moorfields Eye Hospital NHS Foundation Trust, London, UK

3 Medignition Inc., Zurich, Switzerland

4 NIHR Biomedical Research Centre at Moorfields Eye Hospital NHS Foundation Trust and UCL Institute of Ophthalmology, London, UK

5 Department of Ophthalmology, Academic Medical Center, Amsterdam, The Netherlands

\section{Introduction}

The recently introduced optical coherence tomography angiography (OCTA) technology has been proposed as a game changer for the detection and monitoring of various chorio-retinal diseases, including age-related macular degeneration (AMD) and diabetic retinopathy (DR) [1]. The OCTA assesses structural and functional information of the retinal and choroidal circulation in a non-invasive manner, thereby providing data that otherwise requires two additional tests: the invasive indocyanine green angiography (ICGA) and fundus fluorescein angiography (FFA). In addition, OCTA allows a depth-resolved assessment of vascular characteristics within individual plexus (superficial and deep capillary plexus, choriocapillaris) and retinal segments (inner and outer retina) in chorio-retinal pathologies.

Today, invasive technologies such as FFA or ICGA are still considered the gold standard for the detection of vascular characteristics associated with chorio-retinal 
diseases [2-4], despite that repeated use is limited due to the risk of adverse events (i.e., allergic reactions) and contraindications, as well as time and cost expenses [5-7]. These downsides triggered the desire of a quick, noninvasive test to replace invasive time- and labor-intensive imaging techniques.

Broad application of OCTA technology is not yet a standard in daily medical routine for several reasons. The analysis of these images is time-consuming and sometimes even requires manual segmentation in some cases. Other disadvantages of OCTA include the limited field of view, the inability to depict leakage and sub-threshold blood flow, and the occurrence of movement and shadowing artifacts. Since 2013 several groups have investigated the diagnostic properties of OCTA. However, the current body of evidence is highly fragmented, scattered, and not easy to access due to inconsistent indexing in electronic databases. We are unaware of any systematic review presenting and summarizing the diagnostic value of OCTA in assessing vascular characteristics in chorio-retinal diseases. In this paper, we therefore conducted a comprehensive review investigating the evidence on the potential of OCTA in the diagnostic work-up of chorio-retinal diseases and the extent to which it could replace FFA in clinical routine.

\section{Methods}

This systematic review was performed following the recommendations of the PRISMA statement [8].

\section{Literature search}

We applied our search strategy without application of language restrictions on Web of Science (by citation of reference), Scopus (from inception until June 12, 2017), and MEDLINE (PubMed interface). The applied search strategy is available on request.

\section{Eligibility criteria}

Eligibility criteria were the availability of primary data allowing to calculate test performance characteristics. For an exploratory meta-analysis, we accepted FFA as reference standard classifying absence or presence of choroidal neovascularization $(\mathrm{CNV})$.

\section{Study selection, data extraction, and quality assessment}

We assessed the methodological quality of included publications as proposed by previously published principles [9]. Following recommendations of Whiting et al. [10], we did not use a summary score for ranking purposes. For the quality assessment, we scrutinized methods of patient selection, data collection, descriptions of the OCTA, and the reference test(s). We considered blinding to be present, if the person(s) classifying a vascular characteristic associated with a chorio-retinal disease (reference test) was unaware of the OCTA examination findings (index test) and vice versa. Two of the authors assessed papers and extracted data by a standardized form which is available on request. A senior epidemiologist was consulted when discrepancies occurred.

\section{Statistical analysis}

Contingency tables consisted of true-positive (TP), falsepositive (FP), true-negative (TN), and false-negative (FN) results. Sensitivity was calculated as $\mathrm{TP} /(\mathrm{TP}+\mathrm{FN})$ and specificity was calculated as $\mathrm{TN} /(\mathrm{FP}+\mathrm{TN})$. We used a unified model that was developed for the meta-analysis of diagnostic accuracy studies and plotted summary receiver operating characteristics (ROC) curves [11]. The indication of $95 \%$ prediction and confidence region on the ROC figure provided estimates of average sensitivity and specificity across included studies.

The minimum number of studies to perform a metaanalysis for a specific vascular characteristic was five. Consequently, a meta-analysis was not feasible for retinal vascular parameters of DR (four studies), polypoidal choroidal vasculopathy (PCV) (one study), and detection of CNV activity (two studies).

We calculated likelihood ratios from the estimated pooled sensitivities and specificities and did not pool negative and positive likelihood ratios, following published recommendations [12].

We performed statistical analyses by using the Stata 14.2 statistical software package (StataCorp. 2015, Stata Statistical Software: Release 14; College Station, TX, USA: StataCorp LP).

\section{Results}

\section{Study selection}

After removing duplicates, electronic searches retrieved 1604 records that were screened by title and/or abstract. Subsequently, we excluded 1556 articles since they did not assess diagnostic accuracy of OCTA, contained no original data or did not investigate chorio-retinal diseases. Finally, 44 articles were considered for inclusion and therefore read in full text. For the systematic review, 17 studies fulfilled the inclusion criteria. No further studies were included after screening the science citation index database or reference lists. 
Fig. 1 Study flowchart

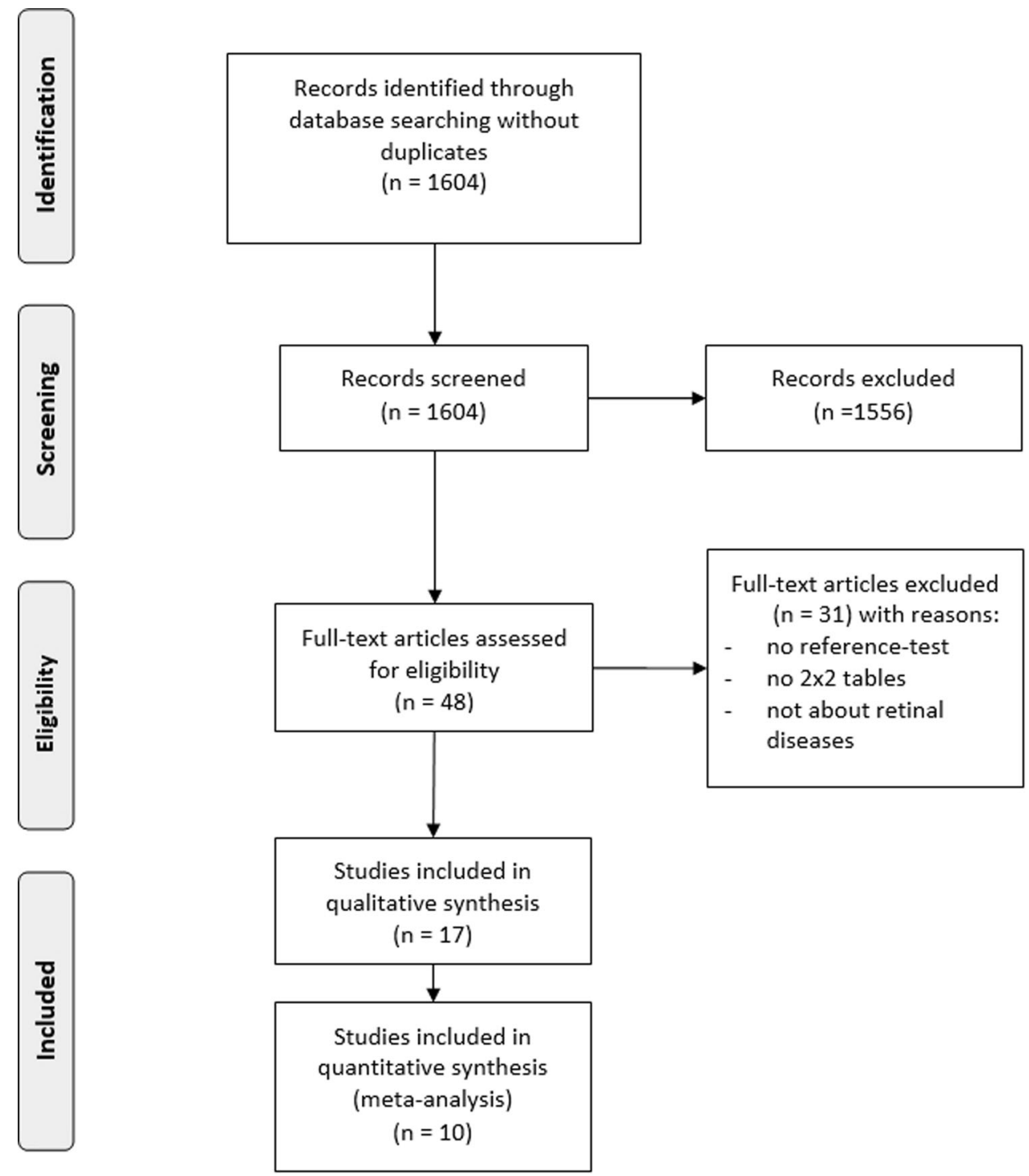

Out of the 17 studies, ten qualified for the inclusion into the meta-analysis [13-22].

We outlined the study selection process in Fig. 1.

\section{Patients' characteristics}

\section{Systematic review}

We included 24 studies, enrolling 19,111 patients and 859,031 images or scans into the systematic review. The study population was heterogeneous regarding the investigated domain and imaging material. Six studies evaluated imaging material of the diagnostic work-up for breast cancer, three looking at mammography, two at ultrasound, and one study at whole-slide images of histopathology associated with central serous chorioretinopathy (CSCR)
$[13,16]$, seven with AMD or PCV [14, 17, 18, 22-25], two with myopia [20, 21], one with foveomacular vitelliform dystrophy (FVD) [19], and one with a mixed cohort of patients [15]. Four studies investigated chorio-retinal vascular characteristics in DR, such as non-perfusion area, vessel density, microaneurysm, and FAZ [4, 26-28].

Among studies investigating eyes with AMD and reporting the proportion of women, percentages ranged from 37.7 to $53.4 \%$.

\section{Meta-analysis}

Ten studies that were included into the exploratory metaanalysis enrolled 440 eyes. The study population was heterogeneous regarding diagnosis, assessed CNV type, and treatment status. Four studies evaluated CNV 
detection in AMD [14, 17, 18, 22], two in CSCR [13, 16], two in myopia [20, 21], one in FVD [19], and one in a mixed cohort of patients [15]. Three studies investigating $\mathrm{CNV}$ in AMD, myopia, and CSCR reported on the proportion of CNV type (in total 77 eyes). Type I was found in 13 eyes (17\%), type II in 45 eyes (58\%), and a mixed type I and II pattern in 19 eyes (25\%) $[13,18,20]$. In those five studies that described previous treatment of CNV, 151 eyes ( $82 \%)$ were treatment naive, 30 eyes were treated by anti-vascular endothelial growth factor (VEGF) (16\%), and 4 eyes $(2 \%)$ had retinal laser treatment.

Among studies investigating CNV detection and reporting the percentage of women ranged from 28.0 to $85.0 \%$ (mean 49.5\%).

We summarized patients' characteristics in Table 1.

\section{Methodological characteristics}

\section{Systematic review}

Of ten cohort studies, three studies were prospective, six retrospective cohorts, and one study did not specify the type of design. Another seven studies (29\%) used a casecontrol design. Within nine out of seventeen studies $(53 \%)$, patients were included in a consecutive manner. Eight studies (47\%) reported on the percentage of eyes that were excluded due to the provision of poor scanning quality and artifacts: the interquartile range was 9.8-20.6\%, the total range reached from $2.7 \%$ to $33.3 \%$ $[4,16-18,20,21,23,25]$.

\section{Meta-analysis}

One study (10\%) investigated a prospectively recruited cohort with a consecutive patient enrollment and five cohort studies were conducted retrospectively. The remaining four studies (40\%) had a case-control design. Overall, seven studies (70\%) enrolled patients in a consecutive manner. Five studies reported on the percentage of eyes that were excluded due to the provision of poor scanning quality and artifacts: the interquartile range was $5.8-12.3 \%$ [16-18, 20, 21].

Methodological characteristics assessed by QUADAS-2 are summarized in Table 2 [10].

\section{Reference tests and index test devices}

\section{Systematic review}

For classification of chorio-retinal vascular characteristics, multimodal imaging (eight studies, 47\%) and FFA alone (six studies, 35\%) were used most often. In all studies multimodal imaging included FFA and/or ICGA, while additional OCT or fundus photography were less frequently used.

In 12 studies (71\%), the AngioVue software was used on the RTVue XR Avanti spectral domain (SD)-OCT device (Optovue, Fremont, CA, USA) to perform OCTA between 2014 and 2015. Other studies performed OCTA on Spectralis OCT (Heidelberg Engineering, Heidelberg, Germany), on AngioPlex CIRRUS HD-OCT model 5000 (Carl Zeiss Meditec Inc., Dublin, USA), or on DRI OCT Triton (Topcon) prototypes. Twelve studies allowed additional manual adaptation of the segmentation boundaries, if necessary $[4,13-15,17-19,21,23-25,28]$.

\section{Meta-analysis}

For classification of chorio-retinal vascular characteristics, multimodal imaging (five studies, 50\%) and FFA alone (five studies, 50\%) were used.

In eight studies (80\%), the AngioVue software was used on the RTVue XR Avanti SD-OCT device (Optovue, Fremont, CA, USA) between 2014 and 2015 to perform OCTA. The six studies that described inner and outer boundaries of outer retinal segmentation reported heterogeneous settings. The inner boundaries were either set on the outer aspect of the inner nuclear layer (INL), at the outer aspect of the outer plexiform layer (OPL), or at the exact level of the OPL. The outer boundaries were either set anterior to or at the exact level of the Bruch's membrane. Within three studies, slabs of the choriocapillaris were also evaluated for CNV detection. Seven studies allowed additional manual adaptation of the segmentation boundaries, if necessary [13-15, 17-19, 21].

Table 3 shows the reference tests that were used for classification.

\section{Test performance}

Ten studies reporting on CNV detection by retinal specialist assessing OCTA images allowed calculating test performance parameters. Sensitivity ranged from 0.50 to 1.00 and specificity ranged from 0.68 to 1.00 . Within the five studies that reported on $\mathrm{CNV}$ detection in AMD sensitivity ranged from 0.50 to 1.00 and specificity ranged from 0.68 to 1.00 . The sensitivity of two studies reporting on the assessment of the FAZ within DR patients ranged from 0.68 to 0.91 and specificity ranged from 0.67 to 0.76 . We provide detailed results in Table 3. Hierarchical summary ROC curves of studies assessing CNV detection are depicted in Fig. 2a across chorio-retinal diseases and in Fig. $2 b$ for AMD. 


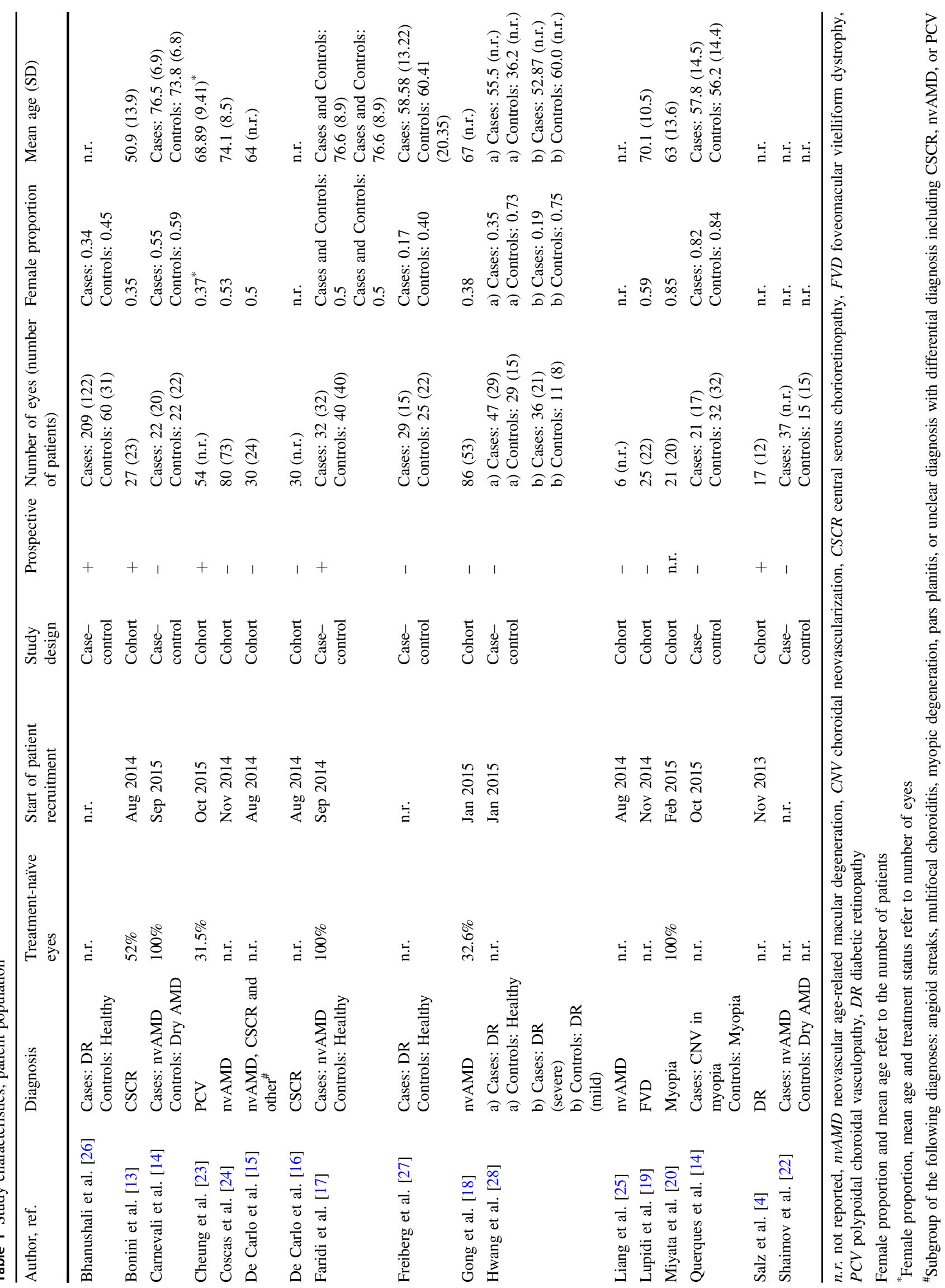


1332

L. Foes et al.

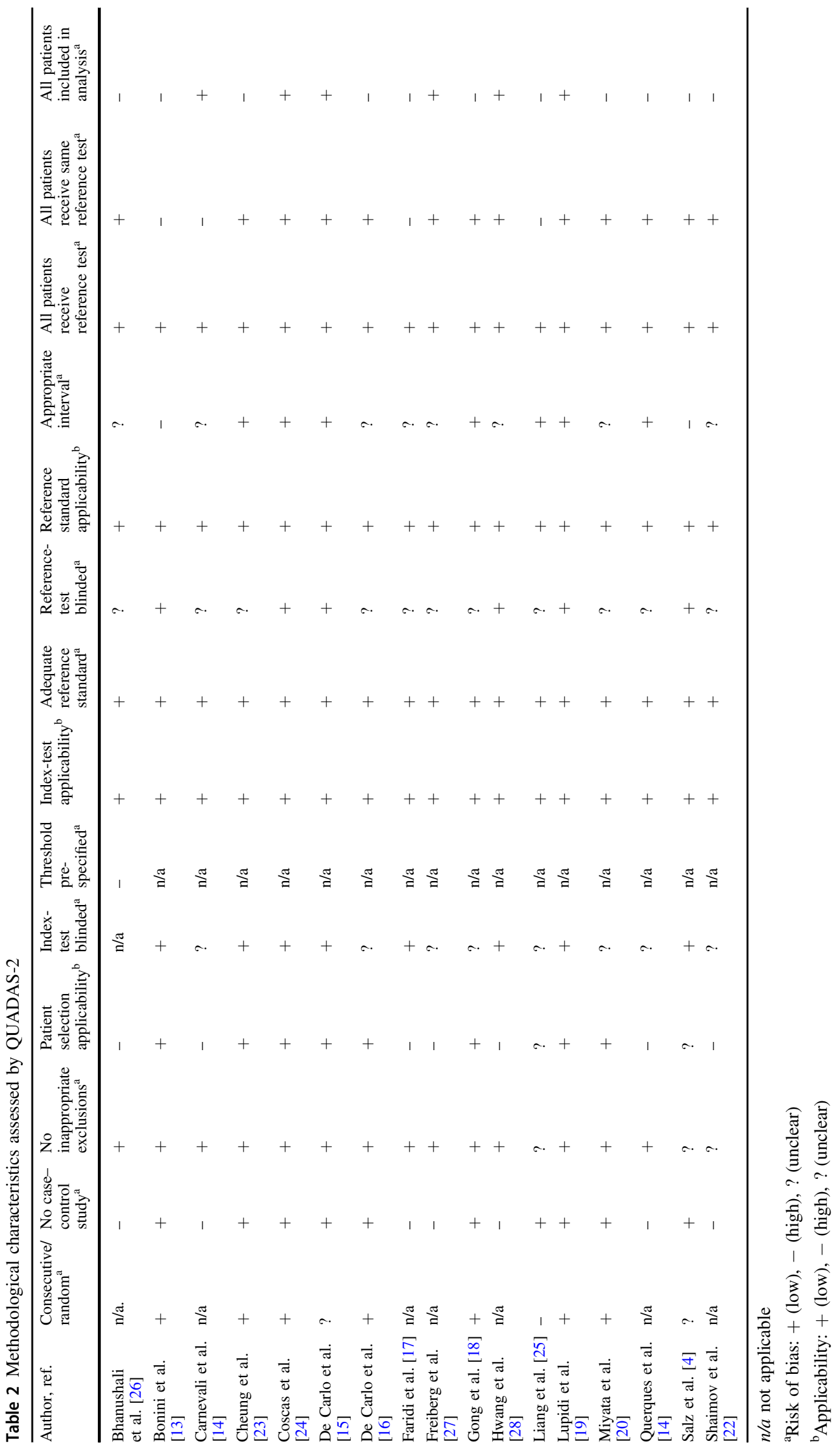

SPRINGER NATURE 


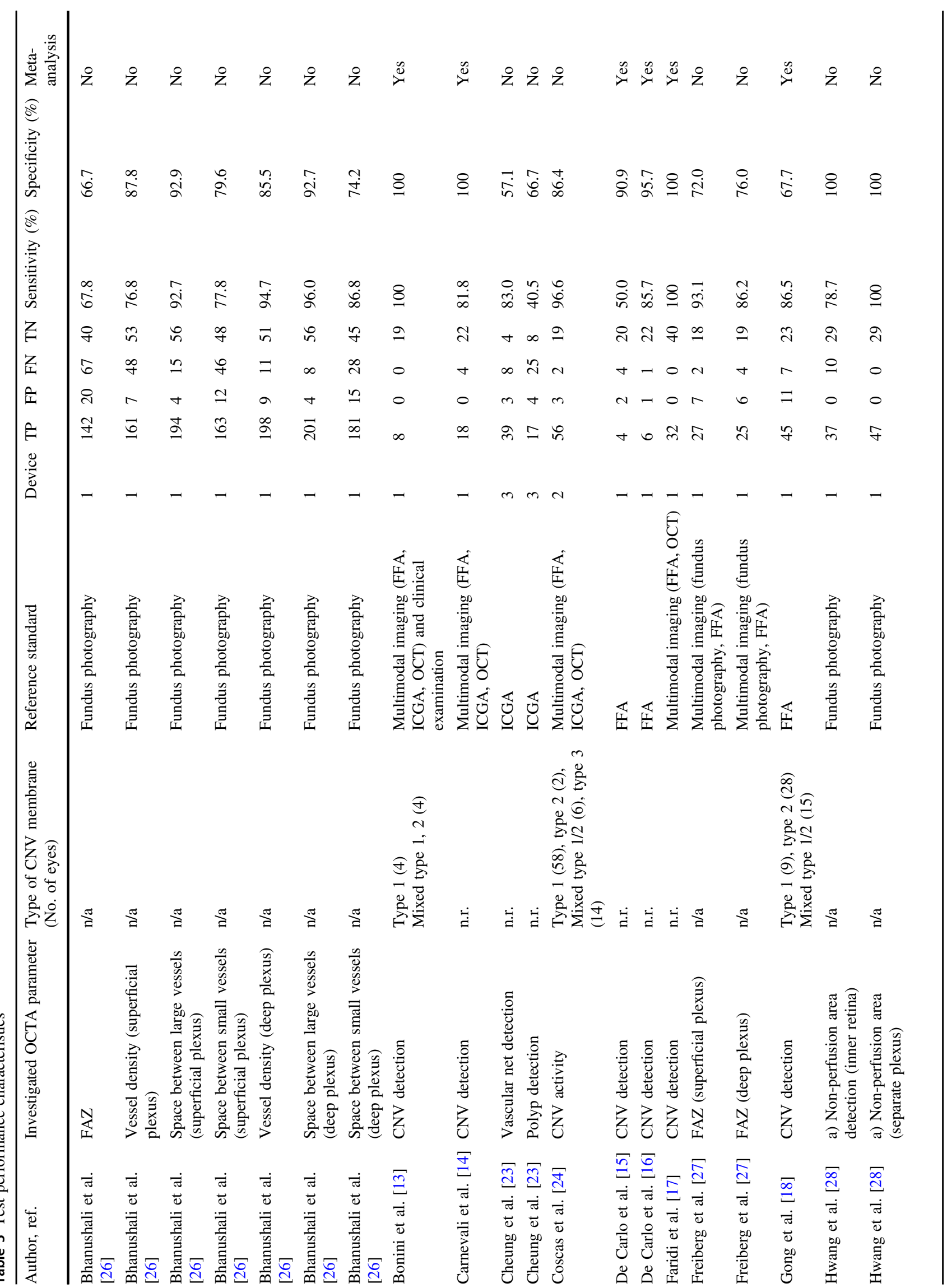




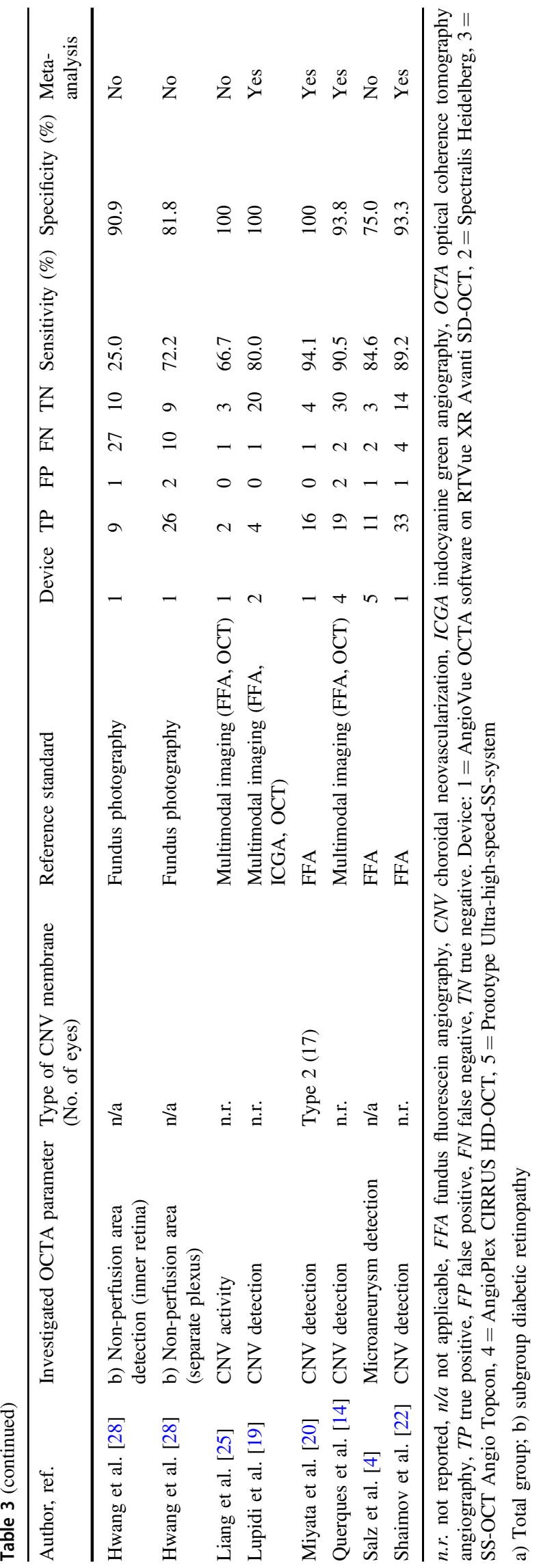

a
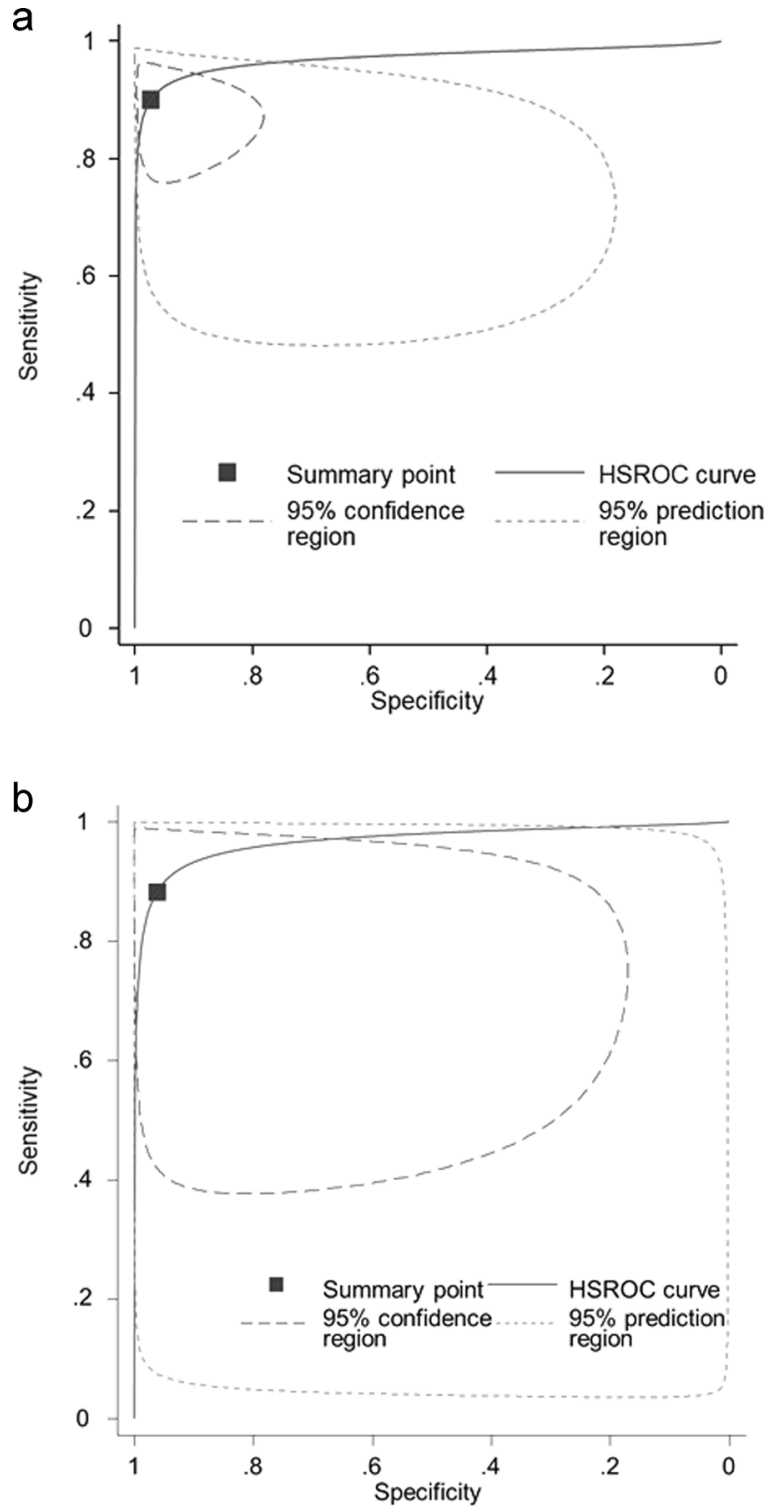

Fig. 2 a Hierarchical summary receiver operating characteristics (ROC) curve of studies assessing choroidal neovascularization (CNV) detection by optical coherence tomography angiography (OCTA). b Hierarchical summary ROC curve of studies assessing CNV detection by OCTA within age-related macular degeneration (AMD) patients

\section{Results from the hierarchical summary ROC analysis}

In general, the pooled sensitivity of studies that assessed CNV detection was 0.90 (95\% confidence intervals (CIs): $0.82-0.95)$ and the pooled specificity was 0.97 (95\% CI: 0.89-0.99). The corresponding positive and negative likelihood ratios were 32.3 (95\% CI: 7.4-141.6) and 0.1 (95\% CI: 0.06-0.20), respectively.

The pooled sensitivity of studies assessing CNV detection in AMD was 0.88 (95\% CI: 0.71-0.96) and specificity was 0.96 (95\% CI: $0.74-1.00)$. The corresponding 
positive and negative likelihood ratios were 22.7 (95\% CI: 2.73-188.2) and 0.12 (95\% CI: 0.04-0.34).

\section{Discussion}

\section{Main findings}

An exploratory meta-analysis of CNV detection in OCTA scans, assessed in a small patient sample provided promising results for both sensitivity and specificity. However, four studies used a diagnostic case-control design, which is appropriate for "proof of concept" evaluations, but has been claimed to exaggerate index-test performance [9]. To perform a meta-analysis for vascular characteristics associated with DR, data were too scarce.

\section{Results in the light of existing literature}

Over the past years, research on OCTA has grown exponentially, but to the best of our knowledge, this is the first quantitative and comprehensive assessment of studies investigating the diagnostic value of OCTA. However, there are several narrative reviews on clinical applications and technological characteristics of OCTA available [29-34].

This systematic review shows promising results of OCTA, but it remains unclear to what degree the reported diagnostic accuracies of the heterogeneous and highly biased studies can be transferred into clinical practice. In a personal communication with one of the authors, we learned that some OCTA studies involved many hours of post hoc manual segmentation work, which is not applicable in daily medical routine. Also, it is unknown how uniformly and accurately manual segmentation was performed within the studies included into this systematic review.

Recently, Hwang et al. [28] stated that OCTA could be a promising candidate for monitoring the microvascular status in DR. On the other hand, detection of microaneurysms was shown to be significantly lower compared to FFA. The study by Salz and colleagues [35] corroborated these findings. Interestingly, several authors stated that the OCTA was superior to FFA in the assessment of FAZ. In contrast to FFA, its depth-resolved, non-invasive technology, distinguishes the multiple capillary networks adjacent to the FAZ. Supposedly, the great potential of OCTA currently applies to the assessment of maculopathy, rather than to (peripheral, non-proliferative) retinopathy, due to the limited field of view and the sub-threshold blood flow occurring in microaneurysms. However, the sensitivity of automatic FAZ delineation varied substantially among included studies depending on the investigated plexus and measurement approach [26, 27, 35].

\section{Strength and limitations}

This systematic review applied state of the art methodology [11]. Due to the limited number of studies (and studies per clinical subgroup) separate meta-analyses were not feasible for all clinical strata. For the same reason, we also refrained from exploring heterogeneity statistically. We discovered substantial heterogeneity by clinical (diagnosis, assessed CNV type or proportion of treatment-naive cases) and methodological variation (the quality of reporting and the used study design) between included studies. Arguably, bias was introduced into our results by mixing effects found in cohorts and case-control studies [9]. Most certainly, heterogeneity was also introduced by variations in hardware and software, that is, variations in segmentation boundaries, occurrence rates of artifacts, and in approaches to automated analyses of chorio-retinal vascular parameters, which was used to perform OCTA (see Supplementary material). Another source of substantial bias must have been introduced by the fact that the investigators were presented with conventional cross-sectional OCT scans alongside en face OCTA images. Particularly in the case of CNV detection, conventional cross-sectional OCT scans would have been highly suggestive of CNV. Since studies included into this systematic review were not designed to provide conclusive and clinically useful results, they did not conduct a priori sample-size calculations, which would be required in diagnostic accuracy studies [36].

We excluded several papers comparing OCTA imaging with established reference tests due to the lack of data allowing the construction of two-by-two tables. In this respect, it may be justified to repeat these analyses when additional data are available. Finally, in view that four out of ten studies included in the meta-analysis used a diagnostic case-control design, we believe that the pooled results must be interpreted very cautiously.

\section{Implications for practice}

To date, OCTA has not yet found its place in clinical practice. The degree to which OCTA will be established and ultimately change practice in the future may be decided by its ability to provide robust information on quantifiable and reliable vascular parameters that are comparable across the devices and software. Automated and standardized segmentation that is highly accurate even in patients suffering from chorio-retinal diseases will be needed to use OCTA in the clinical routine. In this context, it will also be vital to seek consensus on viable terminology of OCTA-associated vascular parameters and segmentation boundaries across different devices and software. Further, standardized protocols allowing a rapid image acquisition in busy clinics, even in patients with poor fixation, are needed. Even though 
the proportion of OCTA images providing sufficient scanning quality for grading was comparable to FFA, this is not yet consistent with real-life clinical experience. In the (multimodal imaging) diagnostic work-up of diabetic patients, OCTA may add value by allowing to (i) screen for subclinical microvascular changes; (ii) monitor retinal ischemia; (iii) predict visual prognosis; or (iv) to distinguish non-proliferative from proliferative disease [37]. Future scanning protocols with higher speed will hopefully also allow high-quality depiction of the retinal periphery.

For its successful adoption in the management of patients with CNV, OCTA may be particularly useful to (i) identify quiescent $\mathrm{CNV}$ in fellow eyes; (ii) inform management decisions in patients with persistent SRF under anti-VEGF therapy; or (iii) to offer an alternate way of monitoring the response to anti-VEGF by visualizing growth and regression patterns of the vascular network itself [38].

It will be crucial to balance the trade-off between high scanning sensitivity and motion artifacts. High sensitivity will be required for detection of $\mathrm{CNV}$ in treatment-naive patients in whom CNVs are often not yet properly arterialized and therefore show unorganized and hardly detectable blood flow. This also applies to patients with large pigment epithelial detachments. Motion artifacts are most often seen in patients with CNV due to poor fixation skills resulting from low visual acuity. Conversely, projection artifacts may come at the heels of incorrectly segmented retinal slabs, that is, mimicking vascular networks in investigated pigment epithelial detachments. In any case, currently, it is vital to check the corresponding crosssectional OCT(A) image for correct segmentation of the retinal slabs under investigation [39].

\section{Implication for further research}

Only recently the OCTA technology has entered clinical ophthalmological practice. This may be one reason for the limited body of evidence assessing the diagnostic usefulness of OCTA. In clinical routine, OCTA is most commonly used in combination with other imaging techniques. As a result, studies are warranted that will investigate these combined approaches and thereby provide more practical data than single-modality outcomes. Studies included into this systematic review had insufficient reporting quality. We call for reasonably sized prospective studies providing information as proposed by the STARD (Standards for Reporting Diagnostic Accuracys) statement. Future studies will also need to study possible sources of heterogeneity, such as varying conventions for segmentation and terminology. OCTA may be a useful case to apply machine learning algorithms to detect those parameters with as strong association with chorio-retinal diseases. However, these studies too will require large samples of validly collected data allowing a sound derivation and validation of these algorithms.

In view that the high number of artifacts in OCTA images lead to a substantial amount of exclusions within per protocol analyzed studies, it will be essential for future studies to define a strategy how to deal with them in the analysis (i.e. sensitivity analysis) and present this procedure in the publication.

\section{Conclusion}

Findings from preliminary and heterogeneous studies provide promising characteristics of test performance for OCTA assessing vascular parameters associated with chorio-retinal diseases. OCTA offers complementary insight to established modalities in the multimodal imaging diagnostic work-up and may therefore add guidance in patient management. Moreover, it is a viable alternative in patients suffering from chorio-retinal disease and allergic reactions to fluorescein. However, it still needs to be established to what extent these promising results will transfer into daily medical routine.

\section{Summary}

\section{What was known before}

- OCTA technology has been proposed as a game changer for the detection and monitoring of various chorioretinal diseases, including AMD and DR.

- Broad application of OCTA technology is not yet standard in daily medical routine for several reasons.

- Disadvantages of OCTA include the limited field of view, the inability to depict leakage, and sub-threshold blood flow and the occurrence of movement and shadowing artifacts.

\section{What this study adds}

- Findings from preliminary and heterogeneous studies provide promising characteristics of test performance for OCTA assessing vascular parameters associated with chorio-retinal diseases.

- OCTA may be a viable alternative in patients suffering from chorio-retinal vascular disease and allergic reactions to fluorescein.

- It will still need to be established to what extent these results transfer to daily medical routine. 
Funding The research program of the Eye Clinic of the Cantonal Hospital Lucerne, funded by Novartis AG to assess the role of OCTA in screening, diagnosis, and prognosis of retinal diseases.

\section{Compliance with ethical standards}

Conflict of interest The authors declare that they have no conflict of interest.

Publisher's note: Springer Nature remains neutral with regard to jurisdictional claims in published maps and institutional affiliations.

\section{References}

1. Optovue. Optovue introduces first software-assisted OCT device to US market; 2014.

2. Kotsolis AI, Killian FA, Ladas ID, Yannuzzi LA. Fluorescein angiography and optical coherence tomography concordance for choroidal neovascularisation in multifocal choroidtis. Br J Ophthalmol. 2010;94:1506-8.

3. Mehta S. Age-related macular degeneration. Prim Care. 2015; 42:377-91.

4. Salz DA, de Carlo TE, Adhi M, Moult E, Choi W, Baumal CR, et al. Select features of diabetic retinopathy on swept-source optical coherence tomographic angiography compared with fluorescein angiography and normal eyes. JAMA Ophthalmol. 2016;134:644-50.

5. Hopeross M, Yannuzzi LA, Gragoudas ES, Guyer DR, Slakter JS, Sorenson JA, et al. Adverse reactions due to indocyanine green. Ophthalmology. 1994;101:529-33.

6. Kwiterovich KA, Maguire MG, Murphy RP, Schachat AP, Bressler NM, Bressler SB. et al. Frequency of adverse systemic reactions after fluorescein angiography. Results of a prospective study. Ophthalmology. 1991;98:1139-42.

7. Lopez-Saez MP, Ordoqui E, Tornero P, Baeza A, Sainza T, Zubeldia JM, et al. Fluorescein-induced allergic reaction. Ann Allergy Asthma Immunol. 1998;81:428-30.

8. Moher D, Liberati A, Tetzlaff J, Altman DG, Group P. Preferred reporting items for systematic reviews and meta-analyses: the PRISMA statement. Int J Surg. 2010;8:336-41.

9. Lijmer JG, Mol BW, Heisterkamp S, Bonsel GJ, Prins MH, van der Meulen JH, et al. Empirical evidence of design-related bias in studies of diagnostic tests. J Am Med Assoc. 1999;282:1061-66.

10. Whiting PF, Rutjes AW, Westwood ME, Mallett S, Deeks JJ, Reitsma JB, et al. QUADAS-2: a revised tool for the quality assessment of diagnostic accuracy studies. Ann Intern Med. 2011;155:529-36.

11. Harbord RM, Whiting P, Sterne JAC, Egger M, Deeks JJ, Shang $\mathrm{AJ}$, et al. An empirical comparison of methods for meta-analysis of diagnostic accuracy showed hierarchical models are necessary. J Clin Epidemiol. 2008;61:1095-1103.

12. Zwinderman AH, Bossuyt PM. We should not pool diagnostic likelihood ratios in systematic reviews. Stat Med. 2008;27:687-97.

13. Bonini Filho MA, de Carlo TE, Ferrara D, Adhi M, Baumal CR, Witkin AJ, et al. Association of choroidal neovascularization and central serous chorioretinopathy with optical coherence tomography angiography. JAMA Ophthalmol. 2015;133:899-906.

14. Carnevali A, Cicinelli MV, Capuano V, Corvi F, Mazzaferro A, Querques L, et al. Optical coherence tomography angiography: a useful tool for diagnosis of treatment-naive quiescent choroidal neovascularization. Am J Ophthalmol. 2016;169:189-98.

15. de Carlo TE, Bonini Filho MA, Chin AT, Adhi M, Ferrara D, Baumal CR, et al. Spectral-domain optical coherence tomography angiography of choroidal neovascularization. Ophthalmology. 2015;122:1228-38.

16. de Carlo TE, Rosenblatt A, Goldstein M, Baumal CR, Loewenstein A, Duker JS. Vascularization of irregular retinal pigment epithelial detachments in chronic central serous chorioretinopathy evaluated with OCT angiography. Ophthalmic Surg Lasers Imaging Retina. 2016;47:128-33.

17. Faridi A, Jia Y, Gao SS, Huang D, Bhavsar KV, Wilson DJ, et al. Sensitivity and specificity of OCT angiography to detect choroidal neovascularization. Ophthalmol Retina. 2017;1:294-303.

18. Gong J, Yu S, Gong Y, Wang F, Sun X. The diagnostic accuracy of optical coherence tomography angiography for neovascular age-related macular degeneration: a comparison with fundus fluorescein angiography. J Ophthalmol. 2016;2016:7521478.

19. Lupidi M, Coscas G, Cagini C, Coscas F. Optical coherence tomography angiography of a choroidal neovascularization in adult onset foveomacular vitelliform dystrophy: pearls and pitfalls. Invest Ophthalmol Vis Sci. 2015;56:7638-45.

20. Miyata M, Ooto S, Hata M, Yamashiro K, Tamura H, AkagiKurashige $\mathrm{Y}$, et al. Detection of myopic choroidal neovascularization using optical coherence tomography angiography. Am J Ophthalmol. 2016;165:108-14.

21. Querques L, Giuffre C, Corvi F, Zucchiatti I, Carnevali A, De Vitis LA, et al. Optical coherence tomography angiography of myopic choroidal neovascularisation. Br J Ophthalmol. 2017; 101:609-15.

22. Shaimov TB, Panova IE, Shaimov RB, Shaimovsmall ACVA, Shai Mova TA, Fomin AV, et al. Optical coherence tomography angiography in the diagnosis of neovascular age-related macular degeneration. Vestn Oftalmol. 2015;131:4-13.

23. Cheung CMG, Yanagi Y, Mohla A, Lee SY, Mathur R, Chan CM, et al. Characterization and differentiation of polypoidal choroidal vasculopathy using swept source optical coherence tomography angiography. Retina. 2017;37:1464-74.

24. Coscas GJ, Lupidi M, Coscas F, Cagini C, Souied EH. Optical coherence tomography angiography versus traditional multimodal imaging in assessing the activity of exudative age-related macular degeneration: a new diagnostic challenge. Retina. 2015;35:2219-28.

25. Liang MC, de Carlo TE, Baumal CR, Reichel E, Waheed NK, Duker JS, et al. Correlation of spectral domain optical coherence tomography angiography and clinical activity in neovascular agerelated macular degeneration. Retina. 2016;36:2265-73.

26. Bhanushali D, Anegondi N, Gadde SGK, Srinivasan P, Chidambara L, Yadav NK, et al. Linking retinal microvasculature features with severity of diabetic retinopathy using optical coherence tomography angiography. Invest Ophthalmol Vis Sci. 2016;57:Oct519-25.

27. Freiberg FJ, Pfau M, Wons J, Wirth MA, Becker MD, Michels S. Optical coherence tomography angiography of the foveal avascular zone in diabetic retinopathy. Graefes Arch Clin Exp Ophthalmol. 2016;254:1051-58.

28. Hwang TS, Zhang M, Bhavsar K, Zhang X, Campbell JP, Lin P, et al. Visualization of 3 distinct retinal plexuses by projectionresolved optical coherence tomography angiography in diabetic retinopathy. JAMA Ophthalmol. 2016;134:1411-19.

29. Chalam KV, Sambhav K. Optical coherence tomography angiography in retinal diseases. J Ophthalmic Vis Res. 2016;11:84-92.

30. de Carlo TE, Romano A, Waheed NK, Duker JS. A review of optical coherence tomography angiography (OCTA). Int J Retina Vitreous. 2015;1:5.

31. Gao SS, Jia Y, Zhang M, Su JP, Liu G, Hwang TS, et al. Optical coherence tomography angiography. Invest Ophthalmol Vis Sci. 2016;57:OCT27-36.

32. Khan HA, Mehmood A, Khan QA, Iqbal F, Rasheed F, Khan N, et al. A major review of optical coherence tomography angiography. Expert Rev Ophthalmol. 2017;12:373-85. 
33. Tan ACS, Tan GS, Denniston AK, Keane PA, Ang M, Milea D, et al. An overview of the clinical applications of optical coherence tomography angiography. Eye (Lond). 2018;32:262-86.

34. Wylegala A, Teper S, Dobrowolski D, Wylegala E. Optical coherence angiography: a review. Medicine (Baltimore). 2016;95:e4907.

35. Couturier A, Mane V, Bonnin S, Erginay A, Massin P, Gaudric A, et al. Capillary plexus anomalies in diabetic retinopathy on optical coherence tomography angiography. Retina. 2015;35:2384-91.

36. Bachmann LM, Puhan MA, ter Riet G, Bossuyt PM. Sample sizes of studies on diagnostic accuracy: literature survey. BMJ. 2006; 332:1127-29.
37. de Carlo TE, Chin AT, Bonini Filho MA, Adhi M, Branchini L, Salz DA, et al. Detection of microvascular changes in eyes of patients with diabetes but not clinical diabetic retinopathy using optical coherence tomography angiography. Retina. 2015; 35:2364-70.

38. Roisman L, Zhang Q, Wang RK, Gregori G, Zhang A, Chen CL, et al. Optical coherence tomography angiography of asymptomatic neovascularization in intermediate age-related macular degeneration. Ophthalmology. 2016;123:1309-19.

39. Spaide RF, Fujimoto JG, Waheed NK. Optical coherence tomography angiography. Retina. 2015;35:2161-62. 